\title{
Modal waves in multiconductor transmission lines by using fundamental matrix response
}

\author{
Julio Ruiz Claeyssen, Daniela de R. Tolfo and Rosemaira Dalcin Copetti
}

\begin{abstract}
The differential equations that model voltage and current for a multiconductor transmission line are written in matrix form. Supposing a time exponential solution through of the modal analysis the modal waves are obtained and solution of a ordinary matrix differential equation, thus determining the amplitude for voltage and current. The modal waves are given in terms of the fundamental matrix solution associated to the ordinary matrix differential equation. The decomposition of the modal waves in forward and backward propagators are used for determine the reflection and transmission matrices for junction in transmission lines. Circulant symmetric transmission lines are discussed, case in that the values for the self-impedance are the same as well as the mutual-impedance values and the same considerations to the admittance matrix. In particular, for these transmission lines are characterized the propagation constants and is observed that the number of multiconductors has effects only on a specific propagation constant. Numerical example of one multiconductor transmission line is presented allowing to observe important aspects of the methodology developed.
\end{abstract}

Keywords: Multiconductor transmission lines, fundamental matrix solution, junction in transmission lines, circulant matrix, impedance and admittance matrices. 


\section{Introduction}

The equations that determine voltage and current in multiconductor transmission lines are frequently presented as two first order matrix differential equations Paul (2008). In this work these two differential equations are written as only one matrix differential equation of same order. This approach allowed obtain the solutions of current and voltage simultaneously. The modal analysis is realized for to determine the solution, modal wave, using the basis generated by fundamental matrix solution associated with matrix differential equation. The basis considered is given in terms of a scalar function that is solution of a scalar initial value problem.

Discontinuities in transmission lines generate reflected and transmitted waves, these waves are associated to incident wave in the discontinuity through a reflection and transmission matrix, respectively. The methodology development in this paper propose to obtain these matrices using the decomposition of the scalar function and consequently of the fundamental matrix solution. The procedure realized decomposes the modal solution in forward and backward waves. The incident and transmitted waves are associated with forward waves while the reflected wave is associated with a backward wave. The junction between two transmission lines with different impedance and admittance parameters is presented and for this discontinuity are determined reflection and transmission matrices providing the reflected and transmission waves in term of incident waves.

Transmission lines whose impedance and admittance matrices are circulant symmetric (circulant pattern) are used in the study of multiphase networks Heydt e Pierre (2016); Amirhosseini e Cheldavi (2003). Here, in particular the case of a polyphase transmission line with the same value for the self-impedance and same consideration for mutual-impedance parameters as well as admittance values are studied using circulant matrix theory Davis (1979). It is observed that such systems are non-defective and that one propagating constant depend upon the two self and mutual impedance and admittance and of the number of conductors while the remaining propagating constants have the same value and they are independent upon the number of conductors.

A example for a multiconductor transmission line whose impedance and admittance matrices are cyclic symmetric is considered, for mutua and self impedance values equals and same consideration for admittance values. The simulation presented allowed to observe the decomposition proposed.

\section{Multiconductor Transmission Lines}

The multiconductor transmission line equations comprising $n+1$ parallel conductors, 0 being the reference conductor following Paul (2008) are

$$
\begin{aligned}
& \mathcal{L} \frac{\partial \mathbf{I}(t, z)}{\partial t}+\frac{\partial \mathbf{V}(t, z)}{\partial z}+\mathcal{R} \mathbf{I}(t, z)=0 \\
& \mathcal{C} \frac{\partial \mathbf{V}(t, z)}{\partial t}+\frac{\partial \mathbf{I}(t, z)}{\partial z}+\mathcal{G} \mathbf{V}(t, z)=0
\end{aligned}
$$

where

$$
\mathbf{V}(t, z)=\left(\begin{array}{c}
v_{1}(t, z) \\
v_{2}(t, z) \\
\vdots \\
v_{n}(t, z)
\end{array}\right), \quad \mathbf{I}(t, z)=\left(\begin{array}{c}
i_{1}(t, z) \\
i_{2}(t, z) \\
\vdots \\
i_{n}(t, z)
\end{array}\right)
$$

with the components $v_{k}(t, z)$ and $i_{k}(t, k)$ being the voltages and currents at each of the $k$-th line, respectively. The coefficients $\mathcal{L}=\left[L_{i j}\right], \mathcal{R}=\left[R_{i j}\right], \mathcal{C}=\left[C_{i j}\right]$ and $\mathcal{G}=\left[G_{i j}\right]$ are $n \times n$ constant parameter matrices representing the measured per-unit-length inductance $(\mathrm{H} / \mathrm{m})$, resistance $(\Omega / \mathrm{m})$, capacitance $(\mathrm{F} / \mathrm{m})$ and conductance $(\mathrm{S} / \mathrm{m})$ between the $\mathrm{i}$-th and j-th conductors, respectively, and 0 in (1) is the null vector $n \times 1$. In the sequel, it is assumed uniform electromagnetic fields along the z-axis and frequency independent per-unit-length parameters.

Alternatively, the multiconductor transmission line equations in matrix form is given by

$$
\mathbf{A} \frac{\partial \mathbf{U}(t, z)}{\partial t}+\mathbf{B} \frac{\partial \mathbf{U}(t, z)}{\partial z}+\mathbf{D} \mathbf{U}(t, z)=0
$$

being the matrix coefficients in block form

$$
\mathbf{A}=\left(\begin{array}{cc}
\mathbf{0} & \mathcal{L} \\
\mathcal{C} & \mathbf{0}
\end{array}\right), \mathbf{B}=\left(\begin{array}{cc}
\mathrm{I} & \mathbf{0} \\
\mathbf{0} & \mathrm{I}
\end{array}\right), \mathbf{D}=\left(\begin{array}{cc}
\mathbf{0} & \mathcal{R} \\
\mathcal{G} & \mathbf{0}
\end{array}\right)
$$

and the solution vector is

$$
\mathbf{U}(t, z)=\left(\begin{array}{c}
\mathbf{V}(t, z) \\
\mathbf{I}(t, z)
\end{array}\right)
$$


The matrices $\mathbf{A}, \mathbf{B}$ and $\mathbf{D}$ are $2 n \times 2 n$, the vector $\mathbf{U}(t, z)$ is $2 n \times 1$, in this moment $\mathrm{I}$ is the identity matrix $n \times n, \mathbf{0}$ in matrices (3) is the null matrix $n \times n$ while 0 in (2) is the null vector $2 n \times 1$. For simplicity going forward I and $\mathbf{0}$ are the identity and null matrices and 0 is the null vector whose order is as appropriate in referred equation.

\subsection{Modal analysis}

In order to obtain modal wave solutions, are considered in (1) solutions of type

$$
\mathbf{V}(t, z)=e^{\lambda t} V(z), \quad \mathbf{I}(t, z)=e^{\lambda t} I(z)
$$

where $V(z)$ and $I(z)$ are the voltage and current vector amplitudes, respectively, and $\lambda$ is the eigenvalue associated. The substitution of (4) in (1) results in the system

$$
\begin{gathered}
\frac{d V(z)}{d z}+(\lambda \mathcal{L}+\mathcal{R}) I(z)=0 \\
\frac{d I(z)}{d z}+(\lambda \mathcal{C}+\mathcal{G}) V(z)=0
\end{gathered}
$$

that in matrix form is

$$
\mathrm{W}^{\prime}(z)+\mathrm{C}(\lambda) \mathrm{W}(z)=0
$$

where

$$
\mathrm{W}(z)=\left(\begin{array}{c}
V(z) \\
I(z)
\end{array}\right), \quad \mathrm{C}(\lambda)=\lambda \mathbf{A}+\mathbf{D}=\left(\begin{array}{cc}
\mathbf{0} & \lambda \mathcal{L}+\mathcal{R} \\
\lambda \mathcal{C}+\mathcal{G} & \mathbf{0}
\end{array}\right)
$$

Following Claeyssen et al. (1999), the general solution of the equation (7) can be given by

$$
\mathrm{W}(z)=\mathbf{h}(z) \mathbf{a}
$$

where a is a $2 n \times 1$ constant vector and $\mathbf{h}(z)$ is the fundamental matrix solution that is solution of the initial value problem

$$
\mathbf{h}^{\prime}(z)+\mathrm{C}(\lambda) \mathbf{h}(z)=\mathbf{0}, \mathbf{h}(0)=\mathrm{I} .
$$

The fundamental matrix solution can be written as

$$
\mathbf{h}(z)=\sum_{l=0}^{2 n-1} d^{(l)}(z) \mathbf{B}_{l}
$$

where the scalar function $d(z)$ satisfies the scalar initial value problem

$$
\begin{aligned}
& c_{0} d^{(2 n)}(z)+c_{2} d^{(2 n-2)}(z)+\cdots+c_{2 n} d(z)=0, \\
& d(0)=0, \cdots, d^{(2 n-2)}(0)=0, c_{0} d^{(2 n-1)}(0)=1,
\end{aligned}
$$

with the $c_{i}$, for $i=0, \ldots, 2 n$ being the coefficients of the characteristic polynomial

$$
P(\gamma)=\operatorname{det}(\gamma \mathrm{I}+\mathrm{C})=\operatorname{det}\left(\gamma^{2} \mathrm{I}-\mathcal{Z} \mathcal{Y}\right)
$$

where $\gamma$ is the propagation constant, $\mathcal{Z}=\lambda \mathcal{L}+\mathcal{R}, \mathcal{Y}=\lambda \mathcal{C}+\mathcal{G}$ are the block local impedance and admittance matrices, respectively, and

$$
\mathbf{B}_{2 k+1}=\left(\begin{array}{cc}
\mathcal{F}_{k} & \mathbf{0} \\
\mathbf{0} & \mathcal{G}_{k}
\end{array}\right), \mathbf{B}_{2 k}=\left(\begin{array}{cc}
\mathbf{0} & \mathcal{Z} \mathcal{G}_{k} \\
\mathcal{Y} \mathcal{F}_{k} & \mathbf{0}
\end{array}\right)
$$

where

$$
\mathcal{F}_{k}=\sum_{i=0}^{n-1-k} c_{2 i}(\mathcal{Z} \mathcal{Y})^{n-i-1-k}, \mathcal{G}_{k}=\sum_{i=0}^{n-1-k} c_{2 i}(\mathcal{Y} \mathcal{Z})^{n-i-1-k}
$$

The scalar function $d(z)$ is given by

$$
d(z)=\sum_{k=1}^{n} \frac{e^{-\gamma_{i} z}}{P^{\prime}\left(-\gamma_{i}\right)}+\sum_{k=1}^{n} \frac{e^{\gamma_{i} z}}{P^{\prime}\left(\gamma_{i}\right)}=\sum_{k=1}^{n} \frac{2 \sinh \left(\gamma_{i} z\right)}{P^{\prime}\left(\gamma_{i}\right)}
$$


for the case in that $\pm \gamma_{i}$ simple roots of (13), for , $i=1,2, \cdots, n$. While for repeated roots

$$
d(z)=\sum_{l=1}^{k} p_{l}(z) e^{-\gamma_{l} z}+q_{l}(z) e^{\gamma_{l} z},
$$

where $p_{l}(z)$ and $q_{l}(k)$ are polynomials of degree $m_{l}-1$ and $k$ is the number different roots $\gamma_{l}$ with algebraic multiplicity $m_{l}$ in according with Heaviside expansion theorem in Carrier et al. (2005)..

Alternatively the matrix form presented in (7) by a first order matrix differential equations the systems of equations can be decoupled in two second order matrix differential equations. By differentiating the modal system (5) and substituting in (6), and similarly differentiating the modal system (6) and substituting in (5) results in

$$
\begin{array}{r}
\frac{d^{2} V(z)}{d z^{2}}-\Gamma_{\mathcal{Z} \mathcal{Y}}^{2} V(z)=0 \\
\frac{d^{2} I(z)}{d z^{2}}-\Gamma_{\mathcal{Y} \mathcal{Z}}^{2} I(z)=0
\end{array}
$$

with $\Gamma_{\mathcal{Z} \mathcal{Y}}^{2}=\mathcal{Z} \mathcal{Y}$ and $\Gamma_{\mathcal{Y Z}}^{2}=\mathcal{Y} \mathcal{Z}$.

Again using the theory developed by Claeyssen et al. (1999), the solutions of the equations (16) and (17) can be given using the fundamental matrix solution for each one of the differential equations

\section{Decomposition of modal waves}

The scalar function $d(z)$ solution of the (12) can be rewritten as

$$
d(z)=d_{+}(z)+d_{-}(z),
$$

the terms $d_{+}(z)$ and $d_{-}(z)$ are the called the forward and backward terms of the function $d(z)$, respectively. For case the simple roots of polynomial characteristic (13), with $d(z)$ given in (14), results

$$
d_{+}(z)=\sum_{k=1}^{n} \frac{e^{-\gamma_{k} z}}{P^{\prime}\left(-\gamma_{k}\right)}, \quad d_{-}(z)=\sum_{k=1}^{n} \frac{e^{\gamma_{k} z}}{P^{\prime}\left(\gamma_{k}\right)} .
$$

For repeated roots

$$
d_{+}(z)=\sum_{l=1}^{k} p_{l}(z) e^{-\gamma_{l} z}, d_{-}(z)=\sum_{l=1}^{k} q_{l}(z) e^{\gamma_{l} z},
$$

where $p_{l}(z)$ and $q_{l}(z)$ of according with (15).

By considering the decomposition of the $d(z)$ given in equation (18) a decomposition is obtained for $\mathbf{h}(z)$ in (10), given by

$$
\mathbf{h}(z)=\mathbf{h}^{+}(z)+\mathbf{h}^{-}(z),
$$

with

$$
\mathbf{h}^{+}(z)=\sum_{l=0}^{2 n-1} d_{+}^{(l)}(z) \mathbf{B}_{l}, \mathbf{h}^{-}(z)=\sum_{l=0}^{2 n-1} d_{-}^{(l)}(z) \mathbf{B}_{l} .
$$

that allowed identify the corresponding forward and backward terms in $\mathbf{h}(z)$. Consequently, the general solution given in equation (8) can be written

$$
\mathrm{W}(z)=\mathrm{W}^{+}(z)+\mathrm{W}^{-}(z)
$$

where the terms of amplitude are

$$
\mathrm{W}^{+}(z)=\mathbf{h}^{+}(z) \mathrm{a}, \mathrm{W}^{-}(z)=\mathbf{h}^{-}(z) \mathrm{a} .
$$

In particular for a transmission line with two conductors and one reference conductor the conductance, inductance, capacitance and resistance matrices turn out constants $G, L, C, R$, and the fundamental matrix solution in (10) is

$$
\mathbf{h}(z)=\left(\begin{array}{cc}
d^{\prime}(z) & -(\lambda L+R) d(z) \\
-(\lambda C+G) d(z) & d^{\prime}(z)
\end{array}\right)=\mathbf{h}^{+}(z)+\mathbf{h}^{-}(z),
$$

where

$$
\mathbf{h}^{+}(z)=\left(\begin{array}{cc}
1 & Z_{0} \\
Y_{0} & 1
\end{array}\right) d_{+}^{\prime}(z), \quad \mathbf{h}^{-}(z)=\left(\begin{array}{cc}
1 & -Z_{0} \\
-Y_{0} & 1
\end{array}\right) d_{-}^{\prime}(z)
$$


being $Z_{0}=\sqrt{\frac{\lambda L+R}{\lambda C+G}}$ and $Y_{0}=\frac{1}{Z_{0}}$ the impedance and admittance characteristics, respectively. The scalar function $d(z)$ is given by

$$
d(z)=\frac{e^{\gamma z}-e^{-\gamma z}}{2 \gamma}=d_{-}(z)+d_{+}(z)
$$

that satisfies

$$
\begin{gathered}
d^{\prime \prime}(z)-(\lambda L+R)(\lambda C+G) d(z)=0 \\
d(0)=0, \quad d^{\prime}(0)=1
\end{gathered}
$$

being $d_{-}(z)=\frac{e^{\gamma z}}{\gamma}$ and $d_{+}(z)=-\frac{e^{-\gamma z}}{\gamma}$, where $\gamma=\sqrt{(\lambda L+R)(\lambda C+G)}$.

Using the decomposition of matrix $\mathbf{h}(z)$ it is possible to express voltage and current separately as

$$
V^{ \pm}(z)=\mathrm{e}_{1}^{T} \mathbf{h}^{ \pm}(z) \mathrm{a}, I^{ \pm}(z)=\mathrm{e}_{2}^{T} \mathbf{h}^{ \pm}(z) \mathrm{a},
$$

where $\mathrm{e}_{1}^{T}=\left(\begin{array}{ll}1 & 0\end{array}\right), \mathrm{e}_{2}^{T}=\left(\begin{array}{ll}0 & 1\end{array}\right)$. From (23) in (21) is obtained the relation between voltage, current and impedance characteristic

$$
\frac{V^{+}(z)}{I^{+}(z)}=Z_{0}, \frac{V^{-}(z)}{I^{-}(z)}=-Z_{0}
$$

\section{Discontinuities in transmission lines - Junction}

By working in the modal domain, impedance methods does not only simplify calculations because voltage and currents through passive devices become relations of algebraic nature in the sinusoidal steady state. They are convenient in dealing with complicated junctions or terminations. In what follows are obtained the matrices related to the reflected and transmitted waves due to an incident wave at a junction of two transmission lines of different impedance and admittance characteristic. For simplicity, we shall restrict ourselves to the case of transmission line with two conductors for which closed-form expressions are common in the literature.

Let us consider a junction at $z=z_{0}$ of two transmission lines with different characteristic impedances, King (1965), represented in Figure 1. The line 1 has characteristic impedance $Z_{01}=\sqrt{\frac{\lambda L_{1}+R_{1}}{\lambda C_{1}+G_{1}}}$ while the line 2 has characteristic impedance $Z_{02}=\sqrt{\frac{\lambda L_{2}+R_{2}}{\lambda C_{2}+G_{2}}}$. Here $L_{i}, R_{i}, C_{i}, G_{i}, i=1,2$, denote the inductance, resistance, capacitance and conductance at each line, respectively.

In what follows, we assume that a traveling incoming wave at a junction generates a reflected and a transmitted wave as illustrated in Figure 1. By using the wave decomposition (20) results that the incident and reflected waves in line 1 are

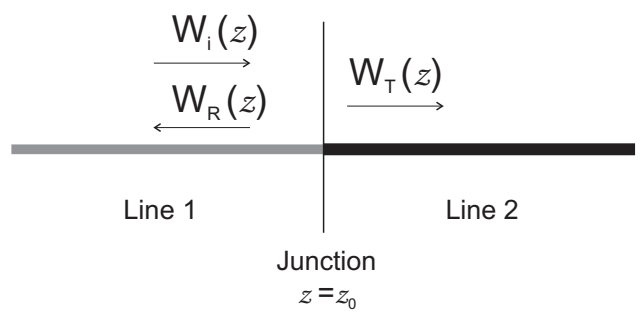

Figura 1: Junction of transmission lines with different impedance and admittance characteristics.

$$
\mathrm{W}_{i}(z)=\mathrm{W}_{1}^{+}(z)=\mathbf{h}_{1}^{+}(z) \mathrm{a}, \quad \mathrm{W}_{R}(z)=\mathrm{W}_{1}^{-}(z)=\mathbf{h}_{1}^{-}(z) \mathrm{a}
$$

respectively, while the transmitted wave in the line 2 is given by

$$
\mathrm{W}_{T}(z)=\mathbf{h}_{2}^{+}(z) \mathbf{b} \text {. }
$$

Here $\mathbf{h}_{1}(z)$ and $\mathbf{h}_{2}(z)$ are the fundamental matrix solutions in line 1 and line 2, respectively, as given in (22). From (23) follows

$$
\mathbf{h}_{1}^{ \pm}(z)=\frac{1}{2} e^{\mp \gamma_{1} z}\left(\begin{array}{cc}
1 & \pm Z_{01} \\
\pm Y_{01} & 1
\end{array}\right), \quad \mathbf{h}_{2}^{+}(z)=\frac{1}{2} e^{-\gamma_{2} z}\left(\begin{array}{cc}
1 & Z_{02} \\
Y_{02} & 1
\end{array}\right)
$$


where $\gamma_{i}=\sqrt{\left(\lambda L_{i}+R_{i}\right)\left(\lambda C_{i}+G_{i}\right)}, i=1,2$. The reflected and transmitted waves are considered proportional to the incident wave by a matrix factor, that is,

$$
\begin{aligned}
\mathbf{W}_{T}\left(z_{0}\right) & =\mathbf{h}_{2}^{+}\left(z_{0}\right) \mathbf{b}=\mathbf{T W}_{i}\left(z_{0}\right), \\
\mathbf{W}_{R}\left(z_{0}\right) & =\mathbf{h}_{1}^{-}\left(z_{0}\right) \mathbf{a}=\mathbf{R W}_{i}\left(z_{0}\right),
\end{aligned}
$$

where $\mathbf{T}$ and $\mathbf{R}$ are transmission and reflection matrices to be determined. The the continuity conditions for the voltage and current at the junction point $z=z_{0}$, are

$$
\mathrm{W}_{i}\left(z_{0}\right)+\mathrm{W}_{R}\left(z_{0}\right)=\mathrm{W}_{T}\left(z_{0}\right)
$$

and the characteristic impedances at each line by (25) are

$$
V_{R}\left(z_{0}\right)+Z_{01} I_{R}\left(z_{0}\right)=0, \quad V_{T}\left(z_{0}\right)-Z_{02} I_{T}\left(z_{0}\right)=0 .
$$

From (26) and (27), the conditions (29) and (30) can be written

$$
\begin{gathered}
\mathbf{h}_{2}^{+}\left(z_{0}\right) \mathbf{b}-\mathbf{h}_{1}^{-}\left(z_{0}\right) \mathbf{a}=\mathbf{h}_{1}^{+}\left(z_{0}\right) \mathbf{a}, \\
\Theta \mathbf{h}_{1}^{-}\left(z_{0}\right) \mathbf{a}=0, \quad \Lambda \mathbf{h}_{2}^{+}\left(z_{0}\right) \mathbf{b}=0
\end{gathered}
$$

where $\Theta$ and $\Lambda$ are the $2 \times 1$ vectors

$$
\Theta=\left(\mathbf{e}_{1}^{T}+Z_{01} \mathrm{e}_{2}^{T}\right)=\left(\begin{array}{ll}
1 & Z_{01}
\end{array}\right), \Lambda=\left(\mathrm{e}_{1}^{T}-Z_{02} \mathrm{e}_{2}^{T}\right)=\left(\begin{array}{ll}
1 & -Z_{02}
\end{array}\right) .
$$

In matrix form, the conditions (31) and (32) for determining the incident and transmitted waves become

$$
\left(\begin{array}{cc}
I & -I \\
\mathbf{0} & -\Theta \\
\Lambda & \mathbf{0}
\end{array}\right)\left(\begin{array}{c}
\mathbf{h}_{2}^{+}\left(z_{0}\right) \mathrm{b} \\
\mathbf{h}_{1}^{-}\left(z_{0}\right) \mathrm{a}
\end{array}\right)=\left(\begin{array}{c}
\mathbf{h}_{1}^{+}\left(z_{0}\right) \mathrm{a} \\
\mathbf{0}
\end{array}\right) .
$$

By solving (33) and considering (26)-(27), the incident and transmitted waves are

$$
\begin{aligned}
\mathbf{W}_{T}\left(z_{0}\right) & =\mathbf{h}_{2}^{+}\left(z_{0}\right) \mathbf{b}=\mathbf{T h}_{1}^{+}\left(z_{0}\right) \mathbf{a}=\mathbf{T W}_{i}\left(z_{0}\right), \\
\mathbf{W}_{R}\left(z_{0}\right) & =\mathbf{h}_{1}^{-}\left(z_{0}\right) \mathbf{a}=\mathbf{R h}_{1}^{+}\left(z_{0}\right) \mathbf{a}=\mathbf{R} \mathbf{W}_{i}\left(z_{0}\right),
\end{aligned}
$$

where

$$
\mathbf{T}=\frac{1}{Z_{02}+Z_{01}}\left(\begin{array}{cc}
Z_{02} & Z_{02} Z_{01} \\
1 & Z_{01}
\end{array}\right), \mathbf{R}=\frac{1}{Z_{02}+Z_{01}}\left(\begin{array}{cc}
-Z_{01} & Z_{02} Z_{01} \\
1 & -Z_{02}
\end{array}\right)
$$

are the transmission and reflection matrices, respectively.

The use of relations (25) allow to obtain $\mathbf{h}^{+}(z)$ a in terms of the voltage or the current as follows

$$
\mathbf{h}^{+}(z) \mathbf{a}=\left(\begin{array}{c}
1 \\
Y_{0}
\end{array}\right) V^{+}(z), \quad \mathbf{h}^{+}(z) \mathbf{a}=\left(\begin{array}{c}
Z_{0} \\
1
\end{array}\right) I^{+}(z) .
$$

In (34) the voltage and current transmitted are linear combination of voltage and current incidents, using (35) these can be rewritten only as multiple of voltage or current incidents. From modes in (34) and with $\mathbf{h}^{+}\left(z_{0}\right)$ a given in (28) and (35) for the characteristic impedance of line 1 , results

$$
\begin{aligned}
\mathrm{W}_{T}\left(z_{0}\right)=\mathbf{h}_{2}^{+}\left(z_{0}\right) \mathbf{b} & =\frac{1}{Z_{01}+Z_{02}}\left(\begin{array}{c}
\left(\begin{array}{cc}
Z_{02} & \left.Z_{02} Z_{01}\right) \mathbf{h}_{1}^{+}\left(z_{0}\right) \mathbf{a} \\
(1 & Z_{01}
\end{array}\right) \mathbf{h}_{1}^{+}\left(z_{0}\right) \mathbf{a}
\end{array}\right) \\
& =\frac{1}{Z_{01}+Z_{02}}\left(\begin{array}{cc}
2 Z_{02} & 0 \\
0 & 2 Z_{01}
\end{array}\right)\left(\begin{array}{c}
V_{1}^{+}\left(z_{0}\right) \\
I_{1}^{+}\left(z_{0}\right)
\end{array}\right),
\end{aligned}
$$

with $V_{1}(z)$ and $I_{1}(z)$ voltage and current for line 1, thus

$$
\mathbf{W}_{T}\left(z_{0}\right)=\mathbf{h}_{2}^{+}\left(z_{0}\right) \mathbf{b}=\mathbf{T}_{J} \mathbf{h}_{1}^{+}\left(z_{0}\right) \mathbf{a}=\mathbf{T}_{J} \mathbf{W}_{i}\left(z_{0}\right),
$$


with $\mathbf{T}_{J}=\left(\begin{array}{cc}T_{V} & 0 \\ 0 & T_{I}\end{array}\right)$, where $T_{V}, T_{I}$ are transmission coefficients for the voltage and current, respectively

$$
T_{V}=\frac{2 Z_{02}}{Z_{02}+Z_{01}}, \quad T_{I}=\frac{2 Z_{01}}{Z_{02}+Z_{01}} .
$$

Analogous reasoning lead us to obtain similar relations for the reflected wave

$$
\mathrm{W}_{R}\left(z_{0}\right)=\mathbf{h}_{1}^{-}\left(z_{0}\right) \mathrm{a}=\mathbf{R}_{J} \mathbf{h}_{1}^{+}\left(z_{0}\right) \mathrm{a}=\mathbf{R}_{J} \mathrm{~W}_{i}\left(z_{0}\right)
$$

with $\mathbf{R}_{J}=\left(\begin{array}{cc}R_{V} & 0 \\ 0 & R_{I}\end{array}\right)$, where $R_{V}, R_{I}$ are the reflection coefficients for the voltage and current

$$
R_{V}=\frac{Z_{02}-Z_{01}}{Z_{02}+Z_{01}}, \quad R_{I}=-R_{V}
$$

Observe that the matrices $\mathbf{T}_{J}$ and $\mathbf{R}_{J}$ are in agreement with (29), once $\mathbf{T}_{J}-\mathbf{R}_{J}=\mathrm{I}$.

\section{Polyphase transmission systems with circulant structure}

Multiconductor transmission lines that have brought the attention in the literature are systems that have a circulatory configuration and circulant symmetric with high phase order, long interconnection and microstrip transmission lines, Heydt e Pierre (2016); Amirhosseini e Cheldavi (2003); Triverio et al. (2010); Dmitriev et al. (2018); Willems (1989); Papaleonidopoulos et al. (2013); Wagenaars et al. (2010); Knockaert et al. (2009); Nitsch et al. (1992), among others. In those systems, impedance and admittance matrices are circulant matrices, Davis (1979), whose pattern has a symmetry insensitive to a circular permutation at its terminals. This kind of matrix structure is amenable for analytical discussion once $N \times N$ circulants can be simultaneously decoupled by using the $N \times N$ Fourier matrix, they commute and the eigenvalues associated with circulants can be simple or repeated and calculated in straigthfoward manner. Thus the modal transformations involved with the diagonalization of circulants can be related to the method of symmetric components due to Fortescue.

In this section, will be discussed the scalar function $d(z)$ for the case of a polyphase network with cyclic symmetry such as N-core cables with common earth screen. The diagonal elements that correspond to the self-impedances in the impedance matrix $\mathcal{Z}$ are assumed to have equal values $Z_{s}$. By assuming cyclic symmetry with respect to the common earth screen, the off-diagonal elements have the same mutual impedances values $Z_{m}$. In Heydt e Pierre (2016), is discussed the case where the mutual impedance is related to the distance between conductors. For the admittance matrix $\mathcal{Y}$ the same considerations are assumed.

Due to its practical importance the case $N=3$ is considered first and we look at the modal analysis for decoupling the matrix $\mathcal{Z Y}$ where

$$
\mathcal{Z}=\left(\begin{array}{ccc}
Z_{s} & Z_{m} & Z_{m} \\
Z_{m} & Z_{s} & Z_{m} \\
Z_{m} & Z_{m} & Z_{s}
\end{array}\right), \mathcal{Y}=\left(\begin{array}{ccc}
Y_{s} & Y_{m} & Y_{m} \\
Y_{m} & Y_{s} & Y_{m} \\
Y_{m} & Y_{m} & Y_{s}
\end{array}\right)
$$

Since $\mathcal{Z}$ and $\mathcal{Y}$ are circulants, the products $\mathcal{Z} \mathcal{Y}, \mathcal{Y} \mathcal{Z}$ are the same once circulants commute, and the also are circulants matrices. The diagonalization of the matrix $\mathcal{Z} \mathcal{Y}$ is given by $F^{*} \Gamma_{Z} \Gamma_{Y} F$, where $F$ is the Fourier matrix, Davis (1979). Moreover their eigenvalues $\alpha=\gamma^{2}$ can be expressed as

$$
\alpha_{k}=\left(Z_{s} Y_{s}+2 Z_{m} Y_{m}\right)+\left(Z_{s} Y_{m}+Z_{m} Y_{s}+Z_{m} Y_{m}\right) w^{k-1}+\left(Z_{s} Y_{m}+Z_{m} Y_{s}+Z_{m} Y_{m}\right) w^{2(k-1)}
$$

with $w=e^{\frac{2 \pi j}{3}}$ or as the product of the eigenvalues of $\mathcal{Z}$ and $\mathcal{Y}$. It turns out that there one simple eigenvalue $\alpha_{1}$ that differ by a factor $\Delta$ from a double eigenvalue $\alpha_{2}=\alpha_{3}$. More precisely,

$$
\begin{gathered}
\alpha_{1}=\left(Z_{s}+2 Z_{m}\right)\left(Y_{s}+2 Y_{m}\right)=\left(Z_{s}-Z_{m}\right)\left(Y_{s}-Y_{m}\right)+\Delta, \\
\alpha_{2}=\left(Z_{s}+Z_{m} w+Z_{m} w^{2}\right)\left(Y_{s}+Y_{m} w+Y_{m} w^{2}\right) \\
=\left(Z_{s}-Z_{m}\right)\left(Y_{s}-Y_{m}\right) \\
\alpha_{3}=\left(Z_{s}+Z_{m} w^{2}+Z_{m} w^{4}\right)\left(Y_{s}+Y_{m} w^{2}+Y_{m} w^{4}\right) \\
=\left(Z_{s}-Z_{m}\right)\left(Y_{s}-Y_{m}\right), \\
\Delta=3 Z_{m} Y_{m}+3 Z_{s} Y_{m}+3 Z_{m} Y_{s} .
\end{gathered}
$$

The triple eigenvalue is obtained when $\Delta=0$. 
Observe that the fact of $\mathcal{Z} \mathcal{Y}=\mathcal{Y} \mathcal{Z}$ implies that the scalar function associated the equations (7), (16) and (17) is the same, thus is possible only consider from (16)-(17) the second-order matrix differential equation

$$
\frac{d^{2} \Phi(z)}{d z^{2}}-\mathcal{Z} \mathcal{Y} \Phi(z)=0
$$

where characteristic polynomial is

$$
P(\gamma)=\operatorname{det}\left(\gamma^{2} \mathbf{I}-\mathcal{Z} \mathcal{Y}\right)
$$

In general, by Davis (1979) for a circulant matrix $\operatorname{circ}\left(c_{1}, c_{2}, \ldots, c_{N}\right)$, the order $N \times N$, has eigenvalues are given by

$$
\alpha_{k}=c_{1}+c_{2} w^{k-1}+\ldots+c_{N}\left(w^{k-1}\right)^{(N-1)}, \quad w=e^{\frac{2 \pi}{N} j}, .
$$

By considering the case of $N \times N$ impedance and admittance matrices with the same characteristics as above, being $N \times N$ symmetric circulants

$$
\mathcal{Z}=\left(\begin{array}{cccc}
Z_{s} & Z_{m} & \cdots & Z_{m} \\
Z_{m} & Z_{s} & \cdots & Z_{m} \\
\vdots & \cdots & \vdots & \vdots \\
Z_{m} & \cdots & Z_{m} & Z_{s}
\end{array}\right), \mathcal{Y}=\left(\begin{array}{cccc}
Y_{s} & Y_{m} & \cdots & Y_{m} \\
Y_{m} & Y_{s} & \cdots & Y_{m} \\
\vdots & \cdots & \vdots & \vdots \\
Y_{m} & \cdots & Y_{m} & Y_{s}
\end{array}\right)
$$

From (37) with $w=e^{\frac{2 \pi j}{N}}$, the eigenvalues $\alpha=\gamma^{2}$ of the circulant matrix $\mathcal{Z} \mathcal{Y}$ are

$$
\begin{aligned}
& \alpha_{1}=\left(Z_{s}+Z_{m}+\ldots+Z_{m}\right)\left(Y_{s}+Y_{m}+\ldots+Y_{m}\right) \\
& \left(Z_{s}-Z_{m}\right)\left(Y_{s}-Y_{m}\right)+\Delta \text {, } \\
& \alpha_{i}=\left(Z_{s}+Z_{m} \frac{\left(w^{i-1}\right)^{n}-w^{i-1}}{w^{i-1}-1}\right)\left(Y_{s}+Y_{m} \frac{\left(w^{i-1}\right)^{n}-w^{i-1}}{w^{i-1}-1}\right) \\
& =\left(Z_{s}-Z_{m}\right)\left(Y_{s}-Y_{m}\right), i=2, \ldots, N \\
& \Delta=N\left(Z_{s} Y_{m}+Z_{m} Y_{s}-2 Z_{m} Y_{m}\right)+N^{2} Z_{m} Y_{m} \text {. }
\end{aligned}
$$

Thus for $\Delta \neq 0$, the characteristic polynomial $P(\gamma)=\operatorname{det}\left(\gamma^{2} I-\mathcal{Z} \mathcal{Y}\right)$ has two simple roots, $\pm \gamma_{1}= \pm \sqrt{\alpha_{1}}$, and two roots $\pm \gamma_{2}= \pm \sqrt{\alpha_{2}}$ with algebraic multiplicity $N-1$. Moreover, for a fixed frequency $\lambda$, the simple root depends upon $N$ and the parameters in the impedance and admittance matrices and the repeated roots are independent of the number of conductors.

Since the characteristic polynomial can be then written as

$$
P(\gamma)=\left(\gamma^{2}-\gamma_{2}^{2}\right)^{N-1}\left(\gamma_{1}^{2}-\gamma_{1}^{2}\right)
$$

the scalar function $d(z)$ can be obtained by Heaviside expansion theorem in Carrier et al. (2005) in according with (15) resulting

$$
d(z)=\sum_{l=1}^{N-1} \frac{\Psi_{l}\left(\gamma_{2}\right)}{(N-1-l) !} z^{N-1-l}\left(e^{\gamma_{2} z}+(-1)^{N+l-2} e^{-\gamma_{2} z}\right)+\frac{1}{P^{\prime}\left(\gamma_{1}\right)} e^{\gamma_{1} z}+\frac{1}{P^{\prime}\left(-\gamma_{1}\right)} e^{-\gamma_{1} z}
$$

where the residues

can be obtained computationally.

$$
\Psi_{l}(\gamma)=\frac{1}{(l-1) !} \frac{d^{(l-1)}}{d \gamma^{(l-1)}}\left(\frac{1}{\left(\gamma+\gamma_{2}\right)^{n-1}\left(\gamma^{2}-\gamma_{1}^{2}\right)}\right)
$$

Observe that although $d(z)$ depends upon a finite number of powers of $z$ due to eigenvalue multiplicity.

\section{Numerical example}

Multiconductor transmission lines with the same mutual and self components in the impedance and admittance matrices has been considered in the literature, in particular the analysis of three-core power cable with common earth screen is realized by Wagenaars et al. (2010) and with equivalent networks in high phase order transmission systems Knockaert et al. (2009); Pandya (2008). Here are presented simulations for a transmission line with $6 \times 6$ impedance and admittance matrices with pattern symmetric circulant. In particular are observed the behavior and decomposition of scalar function $d(z)$ solution of (11)-(12).

For this example, the impedance and admittance matrices are

$$
\mathcal{Z}=\left(\begin{array}{cccccc}
Z_{s} & Z_{m} & Z_{m} & Z_{m} & Z_{m} & Z_{m} \\
Z_{m} & Z_{s} & Z_{m} & Z_{m} & Z_{m} & Z_{m} \\
Z_{m} & Z_{m} & Z_{s} & Z_{m} & Z_{m} & Z_{m} \\
Z_{m} & Z_{m} & Z_{m} & Z_{s} & Z_{m} & Z_{m} \\
Z_{m} & Z_{m} & Z_{m} & Z_{m} & Z_{s} & Z_{m} \\
Z_{m} & Z_{m} & Z_{m} & Z_{m} & Z_{m} & Z_{s}
\end{array}\right), \quad \mathcal{Y}=\left(\begin{array}{cccccc}
Y_{s} & Y_{m} & Y_{m} & Y_{m} & Y_{m} & Y_{m} \\
Y_{m} & Y_{s} & Y_{m} & Y_{m} & Y_{m} & Y_{m} \\
Y_{m} & Y_{m} & Y_{s} & Y_{m} & Y_{m} & Y_{m} \\
Y_{m} & Y_{m} & Y_{m} & Y_{s} & Y_{m} & Y_{m} \\
Y_{m} & Y_{m} & Y_{m} & Y_{m} & Y_{s} & Y_{m} \\
Y_{m} & Y_{m} & Y_{m} & Y_{m} & Y_{m} & Y_{s}
\end{array}\right),
$$




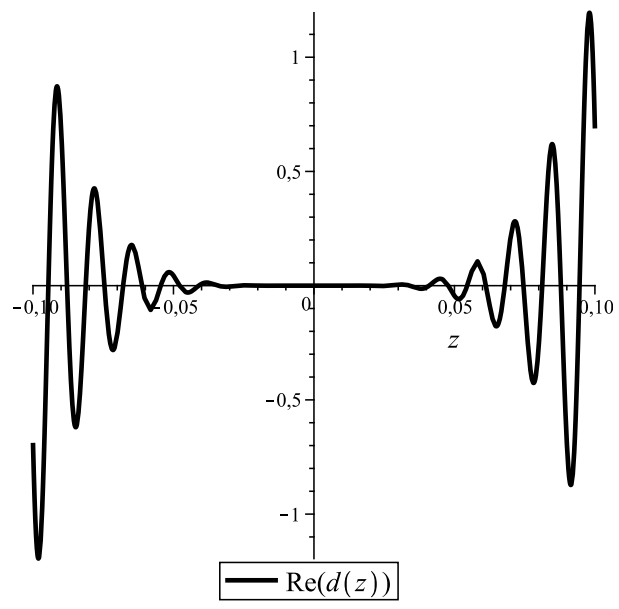

(a) $\operatorname{Re}(d(z))$

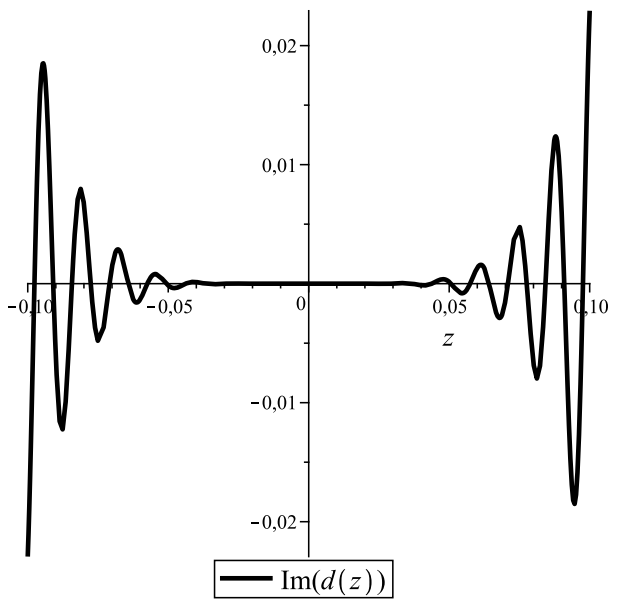

(b) $\operatorname{Im}(d(z))$

Figura 2: Graphics of (a) $\operatorname{Re}(d(z))$ and (b) $\operatorname{Im}(d(z))$.

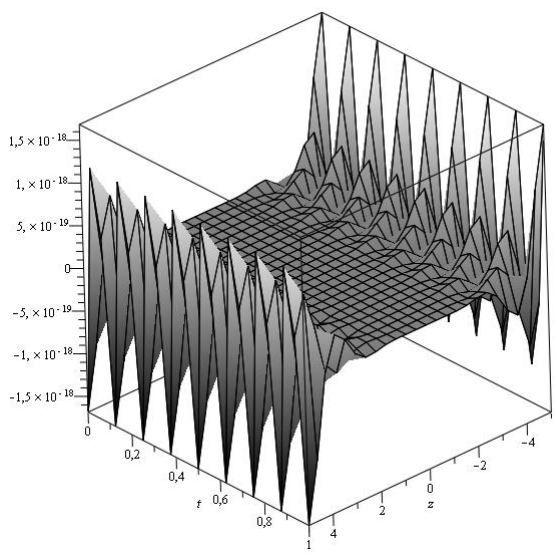

(a) $\operatorname{Re}\left(e^{j \omega t} d(z)\right)$

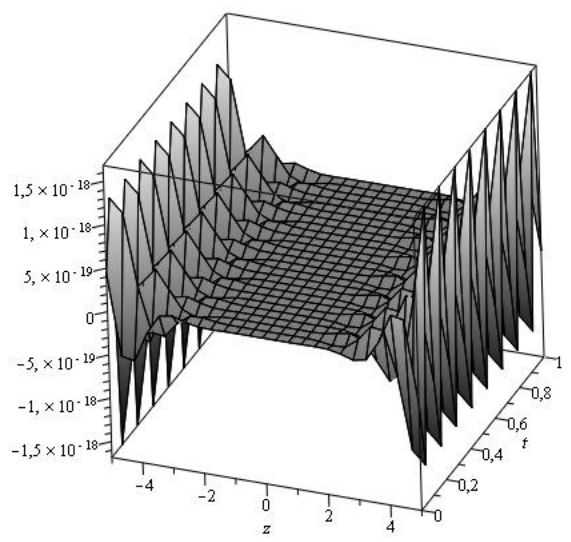

(b) $\operatorname{Im}\left(e^{j \omega t} d(z)\right)$

Figura 3: Graphics of (a) $\operatorname{Re}\left(e^{j \omega t} d(z)\right)$ and (b) $\operatorname{Im}\left(e^{j \omega t} d(z)\right)$.

where $Z_{s}=3.5+j 10^{-6} \omega, Z_{m}=0.35+j 0.11 \cdot 10^{-6} \omega$ and $Y_{s}=10 \cdot 10^{-3}+j 1.5 \cdot 10^{-9} \omega, Y_{m}=-10^{-3}-j 0.07 \cdot 10^{-9} \omega$. The simulations below were performed with $\lambda=j \omega$ and $f=2 \mathrm{GHz}$.

The properties of circulant matrices implies that $\pm \gamma_{k}= \pm \sqrt{\alpha_{k}}$, for $i=1, \ldots, 6$, being $\alpha_{k}$ given in (37). As previously guaranteed, in this case exists two roots quintuples thus the scalar function $d(z)$ has real and imaginary parts in Figure 2(a) and Figure 2(b), respectively, and is composed by combination the functions $z^{4}, z^{3}, z$ and 1 multiplying exponential functions. The function $e^{j \omega t} d(z)$ has real and imaginary parts presented in Figure 3.

Using (19) one has

$$
e^{j \omega t} d_{+}(z)=u_{1}^{+}(t, z)+u_{2}^{+}(t, z)
$$

where $u_{2}^{+}(t, z)=\sum_{k=1}^{5} \phi_{2 k} z^{5-k} e^{-\alpha_{2} z} e^{-j\left(\beta_{2} z-\omega t\right)}, u_{1}^{+}(t, z)=\frac{1}{P^{\prime}\left(-\gamma_{1}\right)} e^{-\alpha_{1} z} e^{-j\left(\beta_{1} z-\omega t\right)}$, the coefficient $\phi_{k}$ can be determined by theoretical Heaviside formula Carrier et al. (2005). Decomposition analogous can be realized with $e^{j \omega t} d_{-}(z)=u_{1}^{-}(t, z)+$ $u_{2}^{-}(t, z)$. The real and imaginary parts of solutions $e^{j \omega t} d_{+}(z)$ and $e^{j \omega t} d_{-}(z)$ are given in Figure 4 and Figure 5 , where is possible observe the oscillation of amplitude and attenuation in direction positive $z$ for $e^{j \omega t} d_{+}(z)$ and negative $z$ for $e^{j \omega t} d-(z)$.

\section{Conclusions}

The use of formulation in matrix form allowed obtain the solution modal simultaneously for voltage and current using as basis the fundamental matrix solution associated to matrix ordinary differential equation. The decomposition of fundamental matrix 


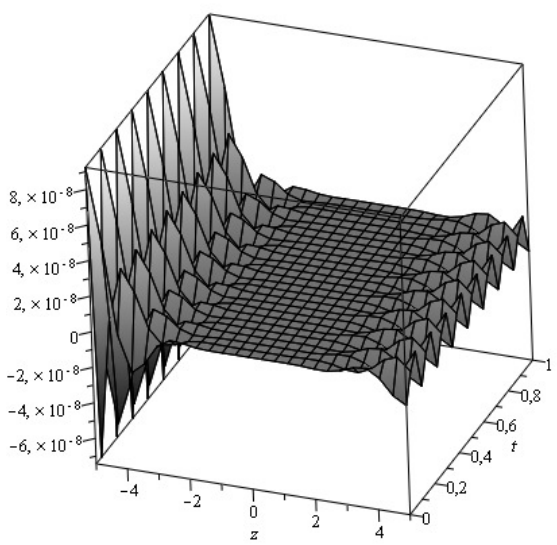

(a) $\operatorname{Re}\left(e^{j \omega t} d_{+}(z)\right)$

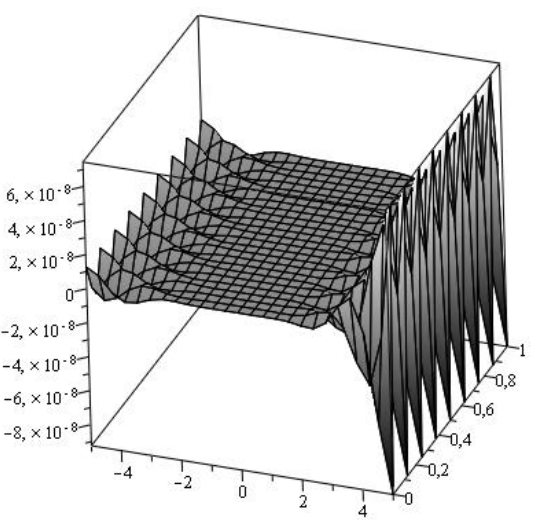

(b) $\operatorname{Re}\left(e^{j \omega t} d_{-}(z)\right.$

Figura 4: Graphics of (a) $\operatorname{Re}\left(e^{j \omega t} d_{+}(z)\right)$ and (b) $\operatorname{Re}\left(e^{j \omega t} d_{-}(z)\right)$.

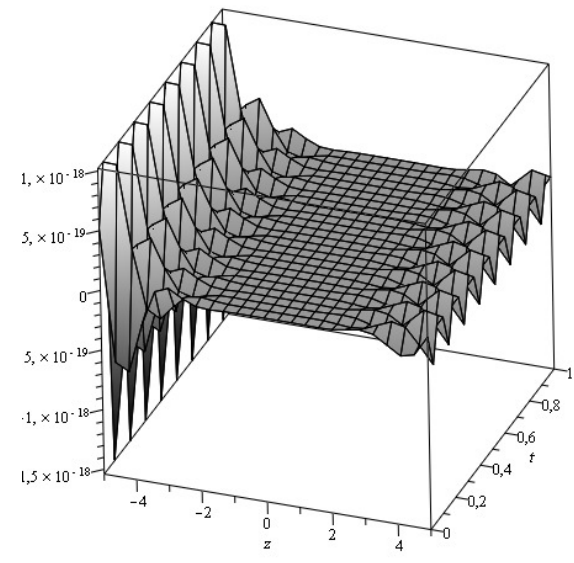

(a) $\operatorname{Im}\left(e^{j \omega t} d_{+}(z)\right)$

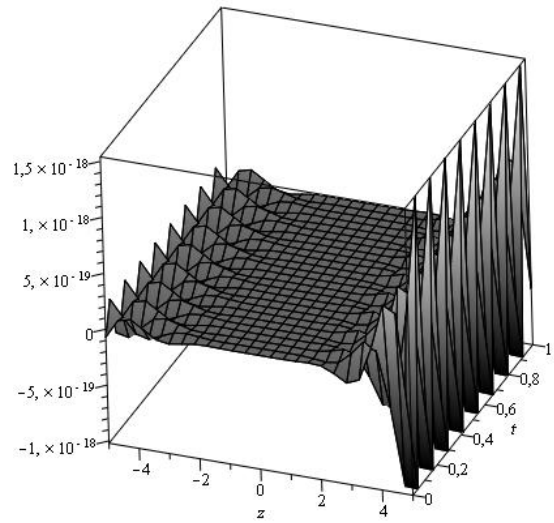

(b) $\operatorname{Im}\left(e^{j \omega t} d_{-}(z)\right.$

Figura 5: Graphics of (a) $\operatorname{Im}\left(e^{j \omega t} d_{+}(z)\right)$ and (b) $\operatorname{Im}\left(e^{j \omega t} d_{-}(z)\right) \ldots$

solution in two waves a one traveling forward and other traveling back is used for determine reflection and transmission matrices in discontinuities as the junction between transmission lines. Multiphase transmission lines with cyclic symmetric pattern are studied with circulant theory depending. Analytical expressions are derived for the eigenvalues of the product of impedance with admittance matrices that have a fixed value for the diagonal elements and for off-diagonal elements another fixed value. It is observed that the number of conductors affect only the propagating structure due to one simple root of a characteristic polynomial. A transmission line with impedance and admittance symmetric circulant matrices of order $6 \times 6$ is presented as numerical example. The obtained graphics for decomposition of the scalar function allowed observe the aspects discussed theoretically in this paper, as repeated propagation constants and different behaviors for decomposition forward and backward waves.

\section{Acknowledgements.}

The first author thanks UFSM/Capes for participating at their Visiting Professor Program. This work was partially supported by Conselho Nacional de Desenvolvimento Científico e Tecnológico (CNPq), 141198/2014 - 1. 


\section{Referências}

Amirhosseini, M. K., Cheldavi, A. (2003). Time domain analysis of circulant symmetric coupled transmission lines. IEE Proc-Microw Antennas Propag, 150(5), 325-331.

Carrier, G. F., Krook, M., Pearson, C. E. (2005). Functions of a Complex Variable: Theory and Thechinique. Society for Industrial and Applied Mathematics.

Claeyssen, J. R., Canahualpa, G., Jung, C. (1999). A direct approach to second-order matrix non-classical vibrating equations. Applied Numerical Mathematics, 30(1), 65-78.

Davis, P. J. (1979). Circulant Matrices. John Wiley \& Sons, Inc., USA.

Dmitriev, V., Portela, G., Martins, L. (2018). Temporal coupled-mode theory of electromagnetic components described by magnetic groups of symmetry. IEEE Transactions on Microwave Theory and Techniques, 66(3), 1165-1171.

Heydt, G. T., Pierre, B. J. (2016). Sequence impedances for high phase order power transmission systems. Em: 2016 IEEE/PES Transmission and Distribution Conference and Exposition, pp. 1-5.

King, R. W. P. (1965). Transmission-line Theory. Dover Publications, Inc..

Knockaert, J., Peuteman, J., Catrysse, J., Belmans, R. (2009). General equations for the characteristic impedance matrix and termination network of multiconductor transmission lines. Em: IEEE International Conference on Industrial Technology, ICIT 2009 , pp. 1-6.

Nitsch, J., Baum, C. E., Sturm., R. (1992). Analytical treatment of circulant nonuniform multiconductor transmission lines. IEEE Transactions on Electromagnetic Compatibility, 34(1), 28-38.

Pandya, A. (2008). Multi-phase power system: performance analysis \& design. Thesis, Doctor of Philosophy in Electrical Engineering, Department of Electrical Engineering, Maharaja Sayajirao University of Baroda.

Papaleonidopoulos, I. C., Theodorou, N. J., Capsalis, C. N. (2013). Travelling-wave modelling of uniform multi-conductor transmission line networks - part i: Analytical derivation. Progress In Electromagnetics Research B, 52, $253-293$.

Paul, C. R. (2008). Analysis of multiconductor transmission lines, $2^{\circ}$ edn. John Wiley \& Sons, Inc..

Triverio, P., Grivet-Talocia, S., Chinea, A. (2010). Identification of highly efficient delay-rational macromodels of long interconnects from tabulated frequency data. IEEE Transactions on Microwave Theory and Techniques, 58(3), 566-577.

Wagenaars, P., Wouters, P. A. A. F., van der Wielen, P. C. J. M., Steennis, E. F. (2010). Measurement of transmission line parameters of three-core power cables with common earth screen. IET Science, Measurement \& Technology, 4(3), 146-155.

Willems, J. L. (1989). A new approach to the analysis of mixed three-phase and six-phase power systems. International Journal of Electrical Power \& Energy Systems, 11(2), 115-122. 